\title{
Perfil nutricional, satisfação com a imagem corporal e erro alimentar em adolescentes com baixo peso
}

\section{Nutritional profile, satisfaction with body image and eating error in adolescents with low weigh}

Aldrin Eder da Silva ${ }^{1}$, Camilla Cristina de Campos Radziavicius²

\section{RESUMO}

OBJETIVO: O objetivo deste estudo é avaliar o perfil nutricional e a satisfação com a imagem corporal de adolescentes com baixo peso e/ou erro alimentar. MÉTODOS: Estudo transversal, com adolescentes atendidos no ambulatório do Instituto de Hebiatria, no Centro Universitário FMABC. RESULTADOS: Vinte e nove adolescentes entre 10 e 18 anos foram incluídos neste estudo (16 mulheres e 13 homens), diagnosticados com baixo peso e/ou erro alimentar. Do total da amostra, 26 adolescentes responderam quanto à satisfação com a imagem corporal; desses, 13 adolescentes $(50,0 \%)$ relataram insatisfação. Três adolescentes $(10,3 \%)$ alcançaram recomendações diárias de consumo de energia. Em relação aos macronutrientes na ingestão diária, 17 adolescentes $(58,6 \%)$ alcançaram recomendações de proteínas, 4 $(13,8 \%)$ atingiram recomendações lipídicas e nenhum atingiu recomendações de carboidratos. Em relação à ingestão de micronutrientes, 5 adolescentes $(17,2 \%)$ alcançaram recomendações diárias de ferro e nenhum dos adolescentes alcançou recomendações de cálcio. CONCLUSÃO: o presente estudo teve como objetivo fornecer observação detalhada sobre adolescentes com baixo peso e / ou erros alimentares. Os resultados evidenciaram hábitos alimentares inadequados, com déficit significativo de nutrientes essenciais, o que pode levar a problemas de saúde. Esta informação é importante para desenvolver melhores estratégias nutricionais para esse público.

Palavras-chave: Perfil Nutricional. Imagem corporal. Baixo peso. Adolescentes.

\section{ABSTRACT}

OBJECTIVE: The objective of this study is to evaluate the nutritional profile and satisfaction with body image of adolescents with low weight and/or eating error. METHODS: Cross-sectional study, with adolescent patients attended in the outpatient service from Institute of Hebiatry, at the Centro Universitário FMABC. RESULTS: Twenty-nine adolescents among 10 and 18 years were enrolled in this study (16 female and 13 male), diagnosed with low weight and/or eating error. Of the total sample, 26 adolescents answered about body image satisfaction, with 13 adolescents $(50,0 \%)$ reported dissatisfaction. Three adolescents $(10.3 \%)$ reached daily energy consumption recommendations. Regarding macronutrients in the daily intake, 17 adolescents $(58.6 \%)$ reached protein recommendations, $4(13.8 \%)$ reached lipid recommendations and none reached carbohydrate recommendations. Regarding micronutrients intake, 5 adolescents (17.2\%) reached daily recommendations of Iron and none of the adolescents reached recommendations of Calcium. CONCLUSION: the present study aimed to provide detailed observation about adolescents with low weight and/or eating errors. The results evidenced inadequate eating habits, with a significant deficit of essential nutrients, which can lead to health issues. This information is important to develop better nutritional strategies for this public.

Keywords: Nutritional Profile. Body image. Low weight. Adol 


\section{INTRODUÇAOO}

A adolescência é o período de transição da infância para a fase adulta, na qual, de acordo com a Organização Mundial de Saúde (OMS), corresponde à faixa cronológica entre 10 e 19 anos. Nesse período, ocorrem importantes transformações biológicas e psicossociais, tendo como resultado intenso crescimento e desenvolvimento (SANTOS et al., 2011). Como parte dessas transformações a imagem corporal do adolescente exerce suma importância nesse período (CORDONI et al., 2016).

A imagem corporal está associada com o desenvolvimento de características sexuais e, assim, inicia-se uma busca pelo corpo desejado. Muitas vezes, para a obtenção de um corpo considerado ideal para o adolescente, ocorre inadimplência quanto ao comportamento alimentar, prejudicando sua percepção da imagem corporal e aumentando os riscos para futuros transtornos alimentares, colocando em risco sua saúde e qualidade de vida (CORDONI et al., 2016). Atualmente, a mídia exerce forte influência na sociedade impondo um padrão de beleza e saúde correspondente a pessoa magra, contribuindo para o aumento da incidência de baixo peso e desnutrição (CASTRO et al., 2010).

A qualidade nutricional nesse contexto se torna fundamental para o desenvolvimento e crescimento ao adolescer, para que haja equilíbrio entre a ingestão e o gasto energético, com equilíbrio entre nutrientes. Esse equilíbrio visa evitar problemas de saúde decorrentes do excesso ou perda de peso, incluindo obesidade, sobrepeso, baixo peso e desnutrição; transtornos alimentares, incluindo a anorexia nervosa, bulimia nervosa e o transtorno da compulsão alimentar periódica, além de outras consequências clínicas como aterosclerose e hipertensão arterial (EISENSTEIN, 2004). A deficiência de ferro (Fe) também tem seu destaque diante de erros alimentares na adolescência, podendo causar anemia e consequentes prejuízos no desenvolvimento neuropsicomotor, cognitivo, déficit no crescimento, apetite e deficiência na resposta do sistema imunológico (GARANITO, 2010).

Dados do Instituto Brasileiro de Geografia e Estatística (IBGE) demonstram a prevalência de déficit de peso, excesso de peso e obesidade no Brasil no período de 20082009 (IBGE, 2009), onde encontramos valores totais de 3,4\% para déficit de peso, 20,5\% para excesso de peso e 4,9\% para obesidade, destacando a importância de intervenções em saúde pública.

Além disso, fatores socioeconômicos, habituais em países menos desenvolvidos e em classes sociais de baixa renda nas quais o acesso à educação e a qualidade nutricional são restritos, estão envolvidos nos problemas de baixo peso e desnutrição (PERES, 2010). 
Dessa forma, crianças e adolescentes apresentam déficits de estatura, cognitivo e psicomotor, apresentando problemas em relação ao crescimento e ao seu desempenho escolar (LEAL et al, 2012). No sexo feminino, é importante destacar os prejuízos quanto à capacidade reprodutiva e à pré-disposição de gerar recém-nascidos com baixo peso (PERES, 2010).

Sendo assim, o objetivo deste projeto é conhecer o perfil nutricional de adolescentes diagnosticados com baixo peso/magreza e/ou erro alimentar atendidos no Instituto de Hebiatria da FMABC, além de identificar sua satisfação com a imagem corporal.

\section{MATERIAIS E METODOS}

O desenho deste projeto é o estudo transversal. A população-alvo constitui-se de pacientes atendidos no Instituto de Hebiatria da FMABC. Os critérios de inclusão são pacientes entre 10 e 19 anos diagnosticados com baixo peso/magreza e/ou erro alimentar. O diagnóstico foi realizado através de análise de informações antropométricas (peso, altura) para cálculo do IMC (Índice de Massa Corporal) (peso/altura²), de acordo com especificação de valores da OMS, conforme a faixa etária e da análise do consumo alimentar através das recomendações do Guia Alimentar para a População Brasileira, do Ministério da Saúde, em que se estabelece a preferência pelo consumo de alimentos in natura ou minimamente processados a alimentos ultraprocessados (MINISTÉRIO DA SAÚDE, 2014).

A seleção da amostra foi definida por conveniência: os pacientes catalogados para o estudo são aqueles que preenchem os critérios de inclusão e que tiveram o primeiro atendimento a partir do início do registro dos dados (agosto de 2017) até fevereiro de 2020.

Como protocolo de estudo, foram coletadas variáveis de identificação (data nascimento, sexo) e dados clínicos como satisfação corporal do paciente. Todos os dados foram obtidos por meio de fichas de anamnese e as informações referentes ao consumo alimentar por meio de recordatórios alimentares de 24 horas obtidos com descrição realizada pelo profissional que atendeu o paciente na primeira consulta de avaliação. Desses recordatórios foram analisados o consumo energético dos macronutrientes carboidratos, proteínas e lipídeos, e oligoelementos $\mathrm{Fe}$ e $\mathrm{Ca}$ utilizando tabelas de composição nutricional de alimentos consumidos no Brasil (TACO e IBGE) (NEPA, 2011; IBGE, 2011), comparando com valores de referência da tabela Dietary Reference Intakes (DRI), na qual constituem recentes revisões e atualizações dos valores de recomendação 
de energia e nutrientes, elaborados à partir de relatórios de comitês especialistas em parceria com o Institute of Medicine dos Estados Unidos e da agência Heath Canada (PADOVANI et al, 2006).

A coleta de dados se deu a partir de revisão dos prontuários dos pacientes, da qual uma base de dados será montada. Os dados foram organizados em planilha do Microsoft Excel®. O programa estatístico para análise de dados é o SPSS versão 23 para Windows. Para os dados sócio-demográficos, a distribuição das variáveis foi descrita como média e desvio padrão ou frequência e proporção, quando aplicável. Para a comparação entre variáveis contínuas, foram utilizados os testes $U$ de Mann-Whitney e o teste t de Student, dependendo das distribuições encontradas. Para as variáveis categóricas, foi utilizado o teste Qui-quadrado de Pearson ou o teste exato de Fisher. Um nível de significância de 5\% $(p \leq 0,05)$ com um nível de confiança de $95 \%$ foi considerado para todos os testes estatísticos.

O protocolo seguiu as condições estabelecidas na Resolução 466/12 do Conselho Nacional de Saúde (CNS). Os dados foram coletados pelo pesquisador de forma anônima e confidencial através de revisão de prontuários e foram arquivadas em local apropriado e não serão usadas para nenhum propósito além dos objetivos propostos no projeto, assim foi solicitado perante ao Comitê de Ética em Pesquisa (CEP) a dispensa do Termo de Consentimento Livre e Esclarecido. O projeto foi aprovado no CEP sob número do Parecer: 3.690.844. CAAE: 20419519.0.0000.0082

\section{RESULTADOS}

Vinte e nove adolescentes entre 10 e 18 anos, diagnosticados com Baixo Peso/ Erro Alimentar, no Instituto de Hebiatria da FMABC, foram incluídos no estudo sendo 16 do gênero feminino e 13 do gênero masculino. Foram revisados os prontuários clínicos e os recordatórios de 24 horas, para análise do perfil nutricional e satisfação com a imagem corporal. Os pacientes possuem média de idade de 14,9 anos (14,8 para meninas e 14,1 para meninos; $p=0,491$ ).

Para diagnóstico de baixo peso dos adolescentes avaliados, utilizou-se o cálculo do IMC (peso/altura²), onde observou-se uma média geral de 16,5 kg/m², uma média de $17,4 \mathrm{~kg} / \mathrm{m}^{2}$ para gênero feminino e $15,5 \mathrm{~kg} / \mathrm{m}^{2}$ para o masculino, com diferença estatisticamente significativa entre os gêneros $(p=0,002)$. A Tabela 1 abaixo apresenta as informações relativas à amostra incluída no estudo. 
Foi questionado aos pacientes da amostra sobre a prática de atividade física. Meninos $(10 ; 76,9 \%)$ relataram realizar mais atividade física do que meninas $(3 ; 18,8 \%)$, também com diferença entre os grupos $(p=0,002)$.

Analisando-se a satisfação com a imagem corporal verificou-se que dos 29 adolescentes observados, 13 não referem satisfação com sua imagem corporal, sem diferença significativa entre os grupos, sendo 9 do gênero feminino e 4 do gênero masculino; 13 referem-se satisfeitos, sendo 6 do gênero feminino e 7 do gênero masculino $(p=0,234)$. Dos 29 adolescentes analisados, 3 não responderam sobre sua satisfação com a imagem corporal, sendo 1 do gênero feminino e 2 do gênero masculino (Tabela 1).

Tabela 1 - Dados demográficos, IMC, atividade física e satisfação com a imagem corporal.

\begin{tabular}{lrrrr}
\hline Variáveis & $\begin{array}{r}\text { Feminino } \\
(\mathbf{n}=\mathbf{1 6})\end{array}$ & $\begin{array}{r}\text { Masculino } \\
(\mathbf{n}=\mathbf{1 3})\end{array}$ & $\begin{array}{r}\text { Total } \\
(\mathbf{n}=\mathbf{2 9})\end{array}$ & $\boldsymbol{P}$ \\
\hline Idade (média, dp) & $14,8(2,6)$ & $14,1(2,2)$ & $14,9(2,5)$ & 0,491 \\
IMC (média, dp) & $17,4(3,5)$ & $15,5(1,0)$ & $16,5(2,8)$ & ${ }^{*} 0,002$ \\
Atividade Física (freq., \%) & & & & ${ }^{*} 0,002$ \\
$\quad$ Sim & $3(18,8 \%)$ & $10(76,9 \%)$ & $13(44,8 \%)$ & \\
$\quad$ Não & $13(81,2 \%)$ & $3(23,1 \%)$ & $16(55,2 \%)$ &
\end{tabular}

Satisfação com Imagem

corporal **

\begin{tabular}{lllll}
$\operatorname{Sim}$ & $6(40,0 \%)$ & $7(63,6 \%)$ & $13(50,0 \%)$ & 0,234 \\
Não & $9(60,0 \%)$ & $4(36,4 \%)$ & $13(50,0 \%)$ & \\
\hline
\end{tabular}

$\mathrm{dp}=$ desvio padrão

${ }^{*} p<0,05$

** Três indivíduos (1 menina e 2 meninos) não responderam a questão

Em relação ao consumo energético total diário obtido através da análise dos recordatórios, observou-se que a quantidade calórica média consumida pelas meninas foi de $1.123,8 \mathrm{kcal} / \mathrm{dia}$ e pelos meninos, de $1.432,9 \mathrm{kcal} / \mathrm{dia}$; tal diferença não se mostrou estatisticamente significativa $(p=0,060)$. Utilizando-se a fórmula de Harris-Benedict para estabelecimento do GET (Gasto Energético Total), verificou-se uma quantidade calórica de $1.589,3 \mathrm{kcal} /$ dia para meninas e $1.827,8 \mathrm{kcal} /$ dia para meninos $(\mathrm{p}=0,013)$. Foram calculados os percentuais relativos ao consumo relatado de acordo com as recomendações diárias; em média, meninas atingiram $71,2 \%$ e meninos, $78,2 \%$ das recomendações diárias do GET; 2 meninas (12,5\% das meninas) e 1 menino (7,7\% dos meninos) atingiram níveis acima do recomendado diário (Tabela 2). De acordo com os alimentos citados nos 
recordatórios, observou-se uma média de 9 alimentos diferentes, sendo 1 alimento correspondente ao grupo das Frutas, 1 alimento para o grupo das Hortaliças, 4 alimentos classificados como Processados e Ultraprocessados de acordo com o Guia Alimentar para a População Brasileira (MINISTÉRIO DA SAÚDE, 2014).

Observando-se o consumo de macronutrientes (Tabela 2), a ingesta diária referida ao grupo de carboidratos foi de 146,8g para meninas e 193,8g para meninos; tal diferença mostrou-se estatisticamente significativa $(p=0,032)$. Meninas atingiram, em média, $62,8 \%$ das recomendações diárias para o consumo desse macronutriente; meninos atingiram 70,5\%. Nenhum indivíduo da amostra atingiu níveis acima do recomendado. Em relação ao consumo de proteínas, a ingesta diária para meninas foi de 47,3g; para meninos, a ingesta foi de 57,5g ( $p=0,207)$. Em média, meninas atingiram 119,3\% das recomendações diárias; para os meninos, este valor foi de $127,7 \%$, mostrando uma tendência de maior ingesta de proteínas. A maioria das meninas (9 meninas; 56,2\%) atingiram níveis acima dos recomendados para ingesta diária desse macronutriente, assim como a maioria dos meninos (8 meninos; 61,5\%). Para o consumo de lipídeos, observou-se uma ingesta média diária de 39,3g para meninas e 47,5g para meninos $(p=0,302)$, sem diferença estatisticamente significativa. O percentual médio atingido para a ingesta de lipídeos foi de $74,6 \%$ para meninas e $78,0 \%$ para meninos; duas meninas (12,5\%) atingiram ingesta acima da recomendada, assim como dois meninos (15,4\%). Ressalta-se que, da quantidade de lipídeos ingerida, 38,9\% corresponde a gorduras saturadas.

Tabela 2- Distribuição do consumo de energia, macronutrientes, ferro e cálcio.

\begin{tabular}{lrrrr}
\hline Variáveis & $\begin{array}{r}\text { Feminino } \\
(\mathbf{n = 1 6})\end{array}$ & $\begin{array}{r}\text { Masculino } \\
(\mathbf{n = 1 3})\end{array}$ & $\begin{array}{r}\text { Total } \\
(\mathbf{n = 2 9})\end{array}$ & $\boldsymbol{P}$ \\
\hline Consumo energético total/ & $\mathbf{1 . 1 2 3 , 8 ( 3 9 3 , 1 )}$ & $\mathbf{1 . 4 3 2 , 9}(\mathbf{4 4 1 , 1 )}$ & $\mathbf{1 . 2 6 2 , 4}(\mathbf{4 3 6 , 6 )}$ & $\mathbf{0 , 0 6 0}$ \\
kcal/dia (média, dp) & & & & \\
GET kcal/dia (média/ dp) & $\mathbf{1 . 5 8 9 , 3 ( 1 8 8 , 7 )}$ & $\mathbf{1 . 8 2 7 , 8 ( 2 6 8 , 2 )}$ & $\mathbf{1 . 6 9 6 , 2}(\mathbf{2 5 3 , 9 )}$ & $\mathbf{0 , 0 1 3}$ \\
$\quad$ Acima Recomendado & $2(12,5 \%)$ & $1(7,7 \%)$ & $3(10,3 \%)$ & \\
\% médio atingido & $71,2 \%$ & $78,2 \%$ & $74,3 \%$ & \\
Carboidratos (g) (média/ dp) & $\mathbf{1 4 6 , 8 ( 4 4 , 3 )}$ & $\mathbf{1 9 3 , 8 ( 6 2 , 2 )}$ & $\mathbf{1 6 7 , 9 ( 5 7 , 2 )}$ & $\mathbf{0 , 0 3 2}$ \\
Acima Recomendado & $0(0 \%)$ & $0(0 \%)$ & $0(0 \%)$ & \\
\% médio atingido & $62,8 \%$ & $70,5 \%$ & & \\
Proteínas (g) (média/ dp) & $\mathbf{4 7 , 3 ( 2 1 , 8 )}$ & $\mathbf{5 7 , 5 ( 2 0 , 5 )}$ & $\mathbf{5 1 , 8 ( 2 1 , 5 )}$ & $\mathbf{0 , 2 0 7}$ \\
Acima Recomendado & $9(56,2 \%)$ & $8(61,5 \%)$ & $17(58,6 \%)$ & \\
\% médio atingido & $119,3 \%$ & $127,7 \%$ & $123,0 \%$ &
\end{tabular}


DOI: 10.18605/2175-7275/cereus.v13n2p218-229
SILVA A.E., RADZIAVICIUS C.C.C.

Perfil nutricional, satisfação com a imagem corporal e erro alimentar em adolescentes com baixo peso

\begin{tabular}{|c|c|c|c|c|}
\hline Lipídeos (g) (média/ dp) & $39,3(23,5)$ & $47,5(18,5)$ & $42,9(21,5)$ & 0,302 \\
\hline Acima Recomendado & $2(12,5 \%)$ & $2(15,4 \%)$ & $4(13,8 \%)$ & \\
\hline \% médio atingido & $74,6 \%$ & $78,0 \%$ & $76,1 \%$ & \\
\hline Ferro (mg) (média/ dp) & $7,0(2,9)$ & $8,1(2,7)$ & $7,5(2,8)$ & 0,310 \\
\hline Acima Recomendado & $1(6,2 \%)$ & $4(30,8 \%)$ & $5(17,2 \%)$ & \\
\hline$\%$ médio atingido & $56,9 \%$ & $88,5 \%$ & $71,0 \%$ & \\
\hline Cálcio (mg) (média/ dp) & $306,0(193,1)$ & $413,1(165,9)$ & $354,0(186,3)$ & 0,120 \\
\hline Acima Recomendado & $0(0 \%)$ & $0(0 \%)$ & $0(0 \%)$ & \\
\hline$\%$ médio atingido & $23,4 \%$ & $32,8 \%$ & $27,6 \%$ & \\
\hline
\end{tabular}

$\mathrm{dp}=$ desvio padrão

Com relação aos micronutrientes analisados, a ingesta diária média de Ferro foi de $7,0 \mathrm{mg}$ para meninas e $8,1 \mathrm{mg}$ para meninos $(p=0,310)$, correspondendo a um percentual médio atingido de 56,9\% para meninas e $88,5 \%$ para meninos. Uma menina $(6,2 \%$ das meninas) e 4 meninos (30,8\% dos meninos) relataram atingir níveis acima do recomendado diário. Em relação ao Cálcio, a ingesta média foi de 306,0mg para meninas e 413,1mg para meninos $(p=0,120)$. Meninas atingiram, em média, um percentual de $23,4 \%$ das recomendações diárias desse micronutriente; meninos atingiram 32,8\%. Nenhum indivíduo da amostra relatou consumo acima do recomendado.

\section{DISCUSSÃO}

$\mathrm{Na}$ adolescência, por se tratar de um período quando se evidenciam o crescimento e o desenvolvimento do indivíduo, é fundamental que sejam atingidas as recomendações energéticas estabelecidas, incluindo os macronutrientes e micronutrientes. Diversos fatores influenciam o consumo alimentar dos adolescentes, tais como: valores socioeconômicos e socioculturais, imagem corporal, dietas da moda, alimentos consumidos fora de casa, geralmente processados e ultraprocessados; assim, devemos analisar com ênfase todos os hábitos alimentares neste período da vida (GARANITO, 2010).

Os adolescentes estão passíveis a deficiências nutricionais por conta das altas recomendações diárias dos nutrientes, devido ao intenso crescimento característico desse ciclo da vida, especialmente de ferro, cálcio, zinco e vitaminas $A, C, D, E$ e vitaminas do complexo B (VEIGA et al., 2013). 
O consumo energético total dos adolescentes analisados no estudo ficou abaixo das recomendações: apenas 10,3\% da amostra atingiram as recomendações diárias, sendo 2 $(12,5 \%)$ meninas e 1 (7,7\%) menino. O total da amostra atingiu em média $74 \%$ das recomendações diárias, assim como no trabalho de KAZAPI et al.'2001, onde de um total de 797 adolescentes, em torno de 50\% apresentaram um consumo abaixo das necessidades energéticas.

Em relação ao gênero, os meninos atingiram suas necessidades energéticas um pouco acima em comparação às meninas: $78,2 \%$ e $71,2 \%$ respectivamente. CARVALHO et al., 2013, realizaram estudo com 33 adolescentes, observando que 75,8\% das meninas atingiram suas necessidades calóricas diárias, se sobrepondo aos meninos onde 60,6\% atingiram suas necessidades calóricas diárias.

Os carboidratos possuem as funções de fornecer e armazenar energia no organismo, além de ser componente das membranas celulares e mediar formas de comunicação intracelular (FERRIER, 2018). Dentre os macronutrientes, o consumo de carboidrato foi o que mais ficou abaixo das necessidades diárias estabelecidas no presente estudo, onde $100 \%$ da amostra não atingiu as recomendações diárias, e destas necessidades diárias foram atingidas em média 66\% dessas recomendações. LEAL et al., 2010, observaram que o consumo de carboidratos foi abaixo do limite inferior da recomendação para $46 \%$ dos 228 adolescentes estudados, e no estudo de CARVALHO et al., 2013, com 33 adolescentes, observou-se que 24 adolescentes obtiveram consumo insuficiente, ressaltando assim a importância de estudos e acompanhamento nutricional nesta faixa etária.

No nosso estudo, a proteína foi o macronutriente no qual mais se atingiu os níveis recomendados. Do total da amostra 9 (56,2\%) meninas e 8 (61,5\%) meninos atingiram as recomendações diárias. As proteínas têm por função possibilitar o crescimento e o desenvolvimento essencial do organismo, além da regeneração tecidual (EISENSTEIN, 2004). Dois outros estudos também observaram um alto consumo de proteínas. KASAPI et al., 2001 e VEIGA et al., 2013, encontraram, 50\% e 40\% respectivamente, de adolescentes com consumo superior ao recomendado. KASAPI et al., 2001, ressalta a ideia de que o consumo excessivo de proteína se deve ao fato da crença de que o nutriente promove aumento de força e massa magra sem a pratica de exercício físico.

Os lipídeos têm como principal função fornecer energia para o corpo e constituir a estrutura das membranas celulares. Além disso, tem funções regulatórias ou de coenzimas, e de controle da homeostase corporal através das prostaglandinas e hormônios esteroides 
(FERRIER, 2018). A análise do consumo de lipídeos no nosso estudo mostrou que foi atingido em média 76\% das recomendações diárias dos adolescentes investigados, sendo que $2(12,5 \%)$ meninas e $2(15,4 \%)$ meninos atingiram as recomendações diárias. Novamente observou-se resultados semelhantes no estudo realizado por KASAPI et al., 2001, onde cerca de $69 \%$ dos adolescentes atingiram suas necessidades lipídicas diárias.

Em relação aos micronutrientes analisados, o ferro se destaca por ser considerado a deficiência mais prevalente no mundo e pelas consequências que provoca no desempenho individual. Esta deficiência causa prejuízos a curto e longo prazo no desenvolvimento neuropsicomotor, no aprendizado, no crescimento e compromete o sistema imunológico, sendo que ligada a um quadro de anemia temos uma diminuição da capacidade de transporte de oxigênio pelo sangue, devido a diminuição da concentração da proteína hemoglobina (GARANITO, 2010). No estudo, em média, 17,2\% dos adolescentes estudados atingiram as recomendações diárias, 1 (6,2\%) menina e 4 (30,8\%) meninos, sendo que foi atingido em média no total da amostra $71 \%$ das recomendações diárias desse micronutriente sendo que entre as meninas o percentual atingido (57\%) foi bem menor comparado aos meninos (88\%). Resultado semelhante, apesar de metodologias diferentes, foram encontrados com o estudo de LEAL et al., 2010, onde $12 \%$ dos meninos tiveram a ingestão de ferro abaixo do recomendado contra 41\% das meninas. BORGES et al., 2009, realizou um estudo para quantificar o percentual de crianças e adolescentes da rede pública de ensino fundamental da cidade de Salvador- BA com anemia e obteve um resultado de 24,5\% de prevalência evidenciando assim o problema de saúde pública no país.

O cálcio exerce papel importante para o crescimento e maturação dos ossos, sendo este desenvolvimento mais intenso na adolescência (LEAL et al., 2010).

Analisando o cálcio, observamos que $100 \%$ da amostra não atingiu a recomendação diária, sendo que foi atingido em média apenas $28 \%$ das necessidades diárias deste micronutriente no presente estudo. Foi o índice mais baixo encontrado entre todos os nutrientes analisados. VEIGA et al., 2013, também encontrou em seu estudo índice preocupante quanto a ingestão do cálcio onde quase 100\% dos 6797 adolescentes investigados tiveram inadequação da ingestão do mineral e LEAL et al., 2010, também encontrou um baixo índice de ingestão de cálcio, onde em média 93\% da amostra teve seu consumo abaixo do recomendado não atingindo ao menos $50 \%$ da recomendação.

A imagem corporal pode ser definida como a percepção que o indivíduo tem do próprio corpo com base nas sensações e vivências ao longo da vida. A recusa dos adolescentes 
em relação a seu corpo está associada à depressão, distúrbios alimentares como anorexia e bulimia por isso a importância de políticas públicas de promoção da saúde e do peso saudável (CASTRO et al., 2010). Dos 29 adolescentes questionados sobre sua satisfação com a imagem corporal, 55,2\% disseram não estar satisfeitos, destes 9 meninas e 7 meninos. Dois estudos similares mostraram resultados semelhantes, MARQUES et al., 2016, avaliou 323 adolescentes onde 64,6\% estavam insatisfeitos com sua imagem corporal, e SANTOS et al., 2011, teve em seu estudo uma amostra composta por 594 estudantes adolescentes onde $61,3 \%$ não estavam satisfeitos.

Este estudo apresenta algumas limitações. Os resultados obtidos no presente estudo partiram da análise de recordatórios de 24 horas. Este método apresenta algumas desvantagens, pois há uma dificuldade em estimar o tamanho das porções consumidas do alimento relatadas nos relatórios e também não considera a variabilidade do consumo alimentar. Além disso, por se tratar de um estudo com adolescentes com baixo peso, há a tendência a uma superestimação por esse grupo de indivíduos durante a avaliação do consumo alimentar (FISBERG, 2009).

\section{CONSIDERAÇOES FINAIS}

Ressalta-se a falta de estudos nas bases de dados bibliográficas para adolescentes com diagnóstico com baixo peso. A maior parte dos estudos analisados foram desenvolvidos através de observação do perfil nutricional de adolescentes sem foco no baixo peso. Dessa forma, adolescentes com sobrepeso/ obesidade se sobressaíam no total da amostra da maioria dos estudos analisados, dificultando a comparação dos dados, porém corroborando com a tendência de semelhança do déficit dos nutrientes analisados. Com o presente estudo, espera-se uma observação mais detalhada nesse segmento de adolescentes com baixo peso, onde evidenciou os hábitos alimentares inadequados, com déficit significante de nutrientes essenciais podendo levar a prejuízos na saúde, e assim, objetivando fornecer mais informações para o desenvolvimento de políticas específicas nutricionais para promoção de saúde.

\section{REFERÉNCIAS}

BORGES C.Q., SILVA R.C.R., ASSIS A.M.O., PINTO E.J., FIACCONE R.L., PINHEIRO S.M.C. Fatores associados à anemia em crianças e adolescentes de escolas públicas de Salvador, Bahia, Brasil. Cad. Saúde Pública, 2009, Rio de Janeiro, 25(4):877-888 
CARVALHO E.G., MATOS L.M., CAVALCANTE A.C.M., ALMEIDA J.Z. Perfil nutricional de adolescentes praticantes de exercício resistido. Rev Bras Promoc Saude. 2013, Fortaleza, 26(4): 489-497

CASTRO I.R.R., LEVY R.B., CARDOSO L.O., PASSOS M.D., SARDINHA L.M.V., TAVARES L.F., DUTRA S.P., MARTINS A. Imagem corporal, estado nutricional e comportamento com relação ao peso entre adolescentes brasileiros. Ciência \& Saúde Coletiva. 2010, 15(Supl. 2):3099-4108

CORDONI J.K., SILVA J.B., REATO L.F.N., BRITO N.C., MISTURA L.P.F. Comportamento Alimentar e o Adolescer. Adolescência \& Saúde. 2016, v.13, supl 1, p. 72-73

EISENSTEIN E., COELHO S.M. Nutrindo a saúde dos adolescentes: considerações práticas. Adolescência \& Saúde. 2004, volume 1 nำ1, p. 18-26

FERRIER D.R. Bioquímica llustrada. Editora Grupo A, Selo Artmed, 2018, $7^{\circ}$ Edição

FISBERG R.M., MARCHIONI D.M.L., COLUCCI A.C.A. Avaliação do consumo alimentar e da ingestão de nutrientes na prática clínica. Arq Bras Endocrinol Metab.. 2009; 53/5

GARANITO M.P., PITTA T.S., CARNEIRO J.D.A. Deficiência de ferro na adolescência. Rev Ematol Hemoter. 2010, vol. 32, supl. 2

IBGE (INSTITUTO BRASILEIRO DE GEOGRÁFIA E ESTATÍSTICA). Disponível em: https://ww2.ibge.gov.br/home/estatistica/populacao/

condicaodevida/of/2008_2009_encaa/defaulttabzip_prev.sht

IBGE. Tabela De Composição Nutricional Dos Alimentos Consumidos No Brasil. 2011, Rio de Janeiro - RJ

KASAPI I.M., DI PIETRO P.F., AVANCINI S.R.P., FREITAS S.F.T., TRAMONTE V.L.C.G. Consumo de energia e macronutrientes por adolescentes de escolas públicas e privadas. Rev. Nutr. 2001, Campinas, 14 (suplemento): 27-33

LEAL G.V.S., PHILIPPI S.T., MATSUDO S.M.M., TOASSA E.C. Consumo alimentar e padrão de refeições de adolescentes, São Paulo, Brasil. Rev Bras Epidemiol, 2010, 13(3): 457-467

LEAL V.S., LIRA P.I.C., MENEZES R.C.E., OLIVEIRA J.S., COSTA E.C., ANDRADE S.L.L.S. Desnutrição e excesso de peso em crianças e adolescentes: uma revisão de estudos brasileiros. Rev Paul Pediatr. 2012;30(3):415-22

MARQUES M.I., PIMENTA J., REIS S., FERREIRA L.M., PERALTA L., SANTOS M.I., SANTOS S., SANTOS E. (In)Satisfação com a imagem corporal na adolescência. Rev de Pediatria do Centro Hosp do Porto. 2016, vol XXV, nº4

MINISTÉRIO DA SAÚDE. Guia Alimentar da População Brasileira. Brasília- DF, 2014,

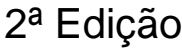


NEPA/ UNICAMP. Tabela Brasileira De Composição De Alimentos (TACO). Campinas - SP, 2011, 4ํㅡㄹ Edição

PADOVANI R.M., AMAYA-FARFÁN A., COLUGNATI F.A.B., DOMENE S.M.A. Dietary reference intakes: aplicabilidade das tabelas em estudos nutricionais. Rev. Nutr. 2006, Campinas, 19(6):741-760

PERES S.V., LATORRE M.R.D.O., SLATER B. Prevalência de Baixo Peso em Adolescentes da Rede de Ensino Público da Cidade de Piracicaba (SP) e seus Fatores Associados. UNOPAR Cient Ciênc Biol Saúde. 2010;12(4):23-8

SANTOS E.M.C., TASSITANO R.F., NASCIMENTO W.M.F., PETRIBÚ M.M.V., CABRAL P.C. Satisfação com o peso corporal e fatores associados em estudantes do ensino médio. Rev Paul Pediatr. 2011, 29(2):214-23.

VEIGA G.V., COSTA R.S., ARAÚJO M.C., SOUZA A.M., BEZERRA I.N., BARBOSA F.S., SICHIERI R., PEREIRA R.A. Inadequação do consumo de nutrientes entre adolescentes brasileiros. Rev. Saúde Pública. 2013, 47(1 Supl):212S-21S 